\title{
Sun-protection habits and knowledge of patients with vitiligo
}

\author{
Leyla Baykal Selçuk ${ }^{1 凶}$, Esma Katkat ${ }^{1}$, Deniz Aksu Arıca ${ }^{1}$, Savaş Yaylıํ, Sevgi Bahadır \\ ${ }^{1}$ Department of Dermatology, Faculty of Medicine, Karadeniz Technical University, Trabzon, Turkey.
}

\begin{abstract}
Introduction: Vitiligo is a common chronic depigmentation disease. Patients are generally advised to protect themselves from the sun.

Methods: One hundred fifty-three patients diagnosed with vitiligo and 106 healthy volunteers with no additional dermatological disease were included in the study. We evaluated the sun-protection habits of patients with vitiligo and controls, and also assessed their knowledge and attitudes toward sun exposure.

Results: Rates of sunscreen use, high-factor sunscreen use, and remaining in the shade were significantly higher among patients $(p=0.004, p=0.028, p=0.040)$. We found significantly higher rates of modifying vacation habits, high sun-protection factors (SPF) sunscreen use, and preferring to remain in the shade among patients using phototherapy $(p=0.016, p=0.019, p=0.028)$. We showed higher rates of modification of vacation habits, wearing sunglasses, and remaining in the shade among patients with longer disease durations ( $p=0.026, p=0.001$, and $p=0.017$ ).

Conclusions: We determined higher rates of sunscreen use, high-SPF sunscreen use, and tendencies to remain in the shade in vitiligo patients compared to the general population. We also determined that disease duration and treatment with phototherapy alter sun-protection habits, but the presence of generalized disease and lesions in visible areas such as the face does not alter patients' habits.
\end{abstract}

Keywords: vitiligo, sun protection, sun exposure, knowledge

Received: 24 September 2019| Returned for modification: 10 November 2019| Accepted: 3 December 2019

\section{Introduction}

Vitiligo is a common chronic depigmentation disease that affects approximately 0.5 to $2 \%$ of the population $(1,2)$. The disease is characterized by autoimmune melanocyte destruction (3). Mechanisms such as genetic, autoimmune, neural, and chemical factors and oxidative damage are particularly implicated in melanocyte destruction. Autoimmune and oxidative stress theories have found the most support in studies $(3,4)$.

One of the basic treatments for vitiligo is phototherapy. There is no consensus on the optimal duration of narrow-band ultraviolet $\mathrm{B}$ (UVB) phototherapy in the treatment of vitiligo (5). An increased risk of skin cancer has been shown with extended psoralen and UVA (PUVA) therapy in psoriasis, but this has not been demonstrated in vitiligo patients. Patients must therefore be aware of the benefits and risks of UV exposure $(6,7)$.

Melanin deficiency in lesional skin in vitiligo patients has been shown to increase photodamage, and exposure to phototherapy is thought to increase the risk of melanoma and non-melanoma skin cancers (NMSC) $(8,9)$. However, another hypothesis is that the autoimmune process in patients protects against the development of melanoma. Paradisi et al. (10) and Teuling et al. (9) have both reported a decreased risk of skin cancer in vitiligo patients. In contrast, Hexsel et al. (7) have reported higher rates of NMSC in vitiligo patients. Another study from Germany observed an increased risk of photodamage and NMSC in patients with vitiligo (11).

Vitiligo patients are generally advised to protect themselves from the sun. However, the number of studies on the knowledge, attitudes, and sun-protection habits of vitiligo patients is very limited. The purpose of this study was to evaluate patients' knowledge, beliefs, and sun-protection habits.

\section{Material and methods}

This cross-sectional study was performed between September 2018 and May 2019 at our dermatology clinic. A multiple-choice questionnaire was prepared by the authors to evaluate the sunprotection habits of patients with vitiligo and controls. Inclusion criteria for cases were a diagnosis with vitiligo at least six months prior and consent to complete the questionnaire. Controls were recruited from people without a history of any dermatological or photosensitive diseases, and they consented to complete the questionnaire. We also evaluated patients' clinical demographics, treatments used, type of disease, and disease duration. The sun-protection habits assessed, as suggested previously $(12,13)$, included the use of sunscreens, wide-brimmed hats, and sunglasses, the types of clothing preferred during the summer, avoiding the sun between 11 am and $4 \mathrm{pm}$ in summer, and length of time spent in the open air in summer. We also evaluated the sun-protection factors (SPF) of sunscreens, reapplication of sunscreens during the day, use of sunscreens when swimming, frequency of reapplication of sunscreens after swimming, and types of clothing preferred when sunbathing or swimming. We also evaluated the history of sunburn in the previous year, family and personal history of skin cancers, sun-avoidance behaviors following diagnosis, and whether patients altered their vacation behaviors.

Patients were asked to respond to six statements in order to assess their knowledge and attitudes toward sun exposure in vitiligo. These were: sun makes my disease more visible, sun exacerbates my disease, sun improves my disease, I must protect myself from sun because of my disease, I have an greater risk of skin cancer because of my disease, and I have a lower risk of sunburn because of my disease. 
Data analysis was performed using SPSS 23.0 software (IBM Corp., Armonk, NY, USA). Basic sociodemographic characteristics of participants were assessed using descriptive statistics as means \pm standard deviation and percentages. The frequencies of individual responses were also measured by number and percentage. Chi-square analysis was used to compare descriptive data. The level of statistical significance was accepted as $p<0.05$.

\section{Results}

The study included 153 patients diagnosed with vitiligo at our clinic between September 2018 and May 2019 and 106 healthy volunteers with no additional dermatological disease. The patients consisted of 78 women and 75 men, with a mean age of $30.14 \pm$ 16.41 years. The control subjects consisted of 65 women and 41 men, with a mean age of $30.84 \pm 13.96$ years. In the patient group, 49 people were younger than 18 and 102 were adults. The mean duration of disease was $7.43 \pm 8.71$ years. Forty-two percent of the patients were receiving active treatment. Focal disease was present in $29 \%$ of patients and generalized disease in $56 \%$. We determined that $65 \%$ of patients protected themselves against the sun, $69 \%$ altered their sun-protection behaviors following diagnosis, and $22 \%$ modified their vacation habits. Patients' clinical characteristics and treatments are summarized in Table 1.

Sun-protection behaviors among the patients and controls are summarized in Table 2. The most common method of protection from the sun among our patients was to remain in the shade $(67 \%)$, followed by sunscreen use $(61 \%)$, and wearing widebrimmed hats (47\%), sunglasses (42\%), and long-sleeved clothing (38\%). Rates of sunscreen use, high-factor sunscreen use, and remaining in the shade were significantly higher among patients than controls ( $p=0.004, p=0.028$, and $p=0.040)$.

Disease severity and sun-protection behaviors are evaluated in Table 3, but no significant differences were determined. When knowledge of and beliefs concerning the disease were analyzed, we observed a high level of belief that UV makes the disease more evident among patients with generalized disease $(p=0.032)$. Sunprotection behavior was not different in patients with facial involvement compared to other regions ( $p>0.05)$.

Analysis of the relation between receiving active treatment and sun-protection behaviors revealed significantly higher use of sunscreen and wide-brimmed hats among subjects receiving active treatment, but no relation with other parameters $(p=0.012$ and $p=0.046)$. No significant relation was observed between knowledge of and beliefs concerning the disease and receipt of treatment $(p>0.05)$.

We also wanted to determine the difference in sun-protection behaviors between children (under 18) and adults (18 and older) in the patient group. An analysis of patients' sun-protection behaviors by age groups revealed significantly higher rates of modifying vacation habits following diagnosis, use of SPF 50 sunscreen, and wearing wide-brimmed hats, sunglasses, and long-sleeved clothing among patients aged over 18 ( $p=0.011, p<0.001, p=0.044$, $p=0.010$, and $p<0.0001$, respectively). Sunscreen use was significantly higher among patients under $18(p=0.019)$.

An analysis of the relation between treatment with phototherapy and sun-protection behaviors revealed significantly higher rates of modifying vacation habits, SPF 50 sunscreen use, and preferring to remain in the shade among treated subjects, but no relation with other parameters $(p=0.016, p=0.019$, and $p=0.028$, respectively). No significant relation was determined between knowledge of and beliefs concerning disease severity and treatment status ( $p>0.05$; Table 4).

Analysis of duration of disease and sun-protection behaviors revealed higher rates of modification of vacation habits, use of sunglasses, and remaining in the shade among subjects with longer disease durations ( $p=0.026, p=0.001$, and $p=0.017$, respectively). Analysis of information and beliefs concerning the disease showed greater belief that sunlight would make the condition more evi-

Table 1 | Characteristics of patients and controls and treatments.

\begin{tabular}{lcc}
\hline Characteristic & $\begin{array}{c}\text { Patients, } \\
n(\%)\end{array}$ & $\begin{array}{c}\text { Controls, } \\
n(\%)\end{array}$ \\
\hline Sex & $78(51)$ & $65(61)$ \\
$\quad$ Female & $75(49)$ & $41(39)$ \\
$\quad$ Male & $30.14 \pm 16.41$ & $30.84 \pm 13.96$ \\
Mean age, year \pm SD & $1(0.65)$ & - \\
History of skin cancer & $16(10.5)$ & $8(8)$ \\
Skin cancer in family & $7.43 \pm 8.71$ & - \\
Disease duration, year \pm SD & & - \\
Type of disease & $45(29)$ & - \\
$\quad$ Focal & $85(56)$ & - \\
$\quad$ Generalized & $23(15)$ & \\
$\quad$ Acrofacial & & \\
Treatment status of vitiligo & $64(42)$ & - \\
$\quad$ Still receiving treatment & & - \\
Therapies & $59(39)$ & - \\
$\quad$ Topical corticosteroid & $99(65)$ & \\
$\quad$ Topical calcineurin inhibitors & $62(41)$ & \\
Phototherapy & $100(65)$ & \\
Use sun protection because of disease & $105(69)$ & \\
Altered sun-protection habits due to & $33(22)$ & \\
vitiligo & & \\
Altered vacation habits since diagnosis & & \\
SD = standard deviation & & \\
\end{tabular}

SD = standard deviation

Table 2 | Sun-protection behaviors of patients and controls.

\begin{tabular}{|c|c|c|c|}
\hline Behavior & $\begin{array}{c}\text { Patients, } \\
n(\%)\end{array}$ & $\begin{array}{c}\text { Controls, } \\
n(\%)\end{array}$ & $p$-value \\
\hline \multicolumn{4}{|l|}{ Use sunscreen } \\
\hline Yes & $90(61)$ & $43(41)$ & \multirow[t]{2}{*}{0.004} \\
\hline No & $63(39)$ & $63(59)$ & \\
\hline \multicolumn{4}{|l|}{ Sunscreen factor } \\
\hline Factor 30 & $13(14)$ & $14(33)$ & \multirow[t]{2}{*}{0.028} \\
\hline Factor 50 & $77(86)$ & $29(67)$ & \\
\hline \multicolumn{4}{|l|}{ Sunscreen reapplication } \\
\hline I reapply it & $28(31)$ & $9(21)$ & \multirow[t]{2}{*}{0.220} \\
\hline I do not reapply it & $62(69)$ & $34(79)$ & \\
\hline \multicolumn{4}{|l|}{ Wear hat on sunny days } \\
\hline Yes & $72(47)$ & $45(42)$ & \multirow[t]{2}{*}{0.464} \\
\hline No & $81(53)$ & $61(58)$ & \\
\hline \multicolumn{4}{|l|}{ Wear sunglasses on sunny days } \\
\hline Yes & $65(42)$ & $33(31)$ & \multirow[t]{2}{*}{0.064} \\
\hline No & $88(58)$ & $73(69)$ & \\
\hline \multicolumn{4}{|l|}{ Hours a day spent in open air } \\
\hline$<5$ hours & $98(64)$ & $70(66)$ & \multirow[t]{3}{*}{0.645} \\
\hline $5-10$ hours & $49(32)$ & $34(32)$ & \\
\hline$>10$ hours & $6(4)$ & $2(2)$ & \\
\hline \multicolumn{4}{|c|}{ Clothing preferred during summer } \\
\hline Short-sleeved or sleeveless & $95(62)$ & $65(61)$ & \multirow[t]{2}{*}{0.900} \\
\hline Long-sleeved & $58(38)$ & $41(39)$ & \\
\hline \multicolumn{4}{|c|}{ Clothing preferred when swimming } \\
\hline Swimsuit & $88(89)$ & $55(79)$ & \multirow[t]{2}{*}{0.106} \\
\hline UV filter clothing & $11(11)$ & $15(21)$ & \\
\hline \multicolumn{4}{|l|}{ Use sunscreen when swimming } \\
\hline Yes & $58(59)$ & $43(61)$ & \multirow[t]{2}{*}{0.710} \\
\hline No & $41(41)$ & $27(39)$ & \\
\hline \multicolumn{4}{|c|}{ Reapply sunscreen after swimming } \\
\hline Yes & $27(27)$ & $26(37)$ & \multirow[t]{2}{*}{0.173} \\
\hline No & $72(73)$ & $44(63)$ & \\
\hline \multicolumn{4}{|c|}{ Avoid sun $11 \mathrm{am}$ to $4 \mathrm{pm}$ in summer } \\
\hline Yes & $100(67)$ & $58(55)$ & \multirow[t]{2}{*}{0.004} \\
\hline No & $50(33)$ & $48(45)$ & \\
\hline
\end{tabular}


dent and that sun protection is essential among subjects with a longer duration of disease ( $p<0.0001$, and $p=0.022$, respectively).

\section{Discussion}

Our findings showed higher prevalence for sunscreen and highfactor sunscreen usage among patients with vitiligo. Patients are more likely to remain in the shade between $11 \mathrm{am}$ and $4 \mathrm{pm}$ in summer than the general population. Rates of sunscreen and wide-brimmed hat use were higher among patients receiving active treatment.

Two previous studies examined sun-protection habits among vitiligo patients. In one of these, Priska et al. (5) retrospectively examined the sun-protection habits of 94 patients with vitiligo. They reported that $71 \%$ of patients used sunscreen and that $36 \%$ preferred SPF 50. The general sunscreen use rates in our study were rather lower, at $61 \%$, although SPF 50 sunscreen use was higher,

Table 3 | Disease severity and sun-protection behaviors.

\begin{tabular}{|c|c|c|c|}
\hline Behavior & $\begin{array}{c}\text { Focal } \\
\text { disease, } \\
n(\%)\end{array}$ & $\begin{array}{c}\text { Generalized } \\
\text { disease, } \\
n(\%)\end{array}$ & $p$-value \\
\hline \multicolumn{4}{|l|}{ Use sunscreen } \\
\hline Yes & $41(60)$ & $49(58)$ & 0.741 \\
\hline No & $27(40)$ & $36(42)$ & \\
\hline \multicolumn{4}{|l|}{ Sunscreen factor } \\
\hline Factor 30 & $9(22)$ & $4(8)$ & 0.121 \\
\hline Factor 50 & $32(78)$ & $45(92)$ & \\
\hline \multicolumn{4}{|l|}{ Sunscreen reapplication } \\
\hline Yes & $12(29)$ & $16(33)$ & 0.730 \\
\hline No & $29(71)$ & $33(67)$ & \\
\hline \multicolumn{4}{|l|}{ Wear hat on sunny days } \\
\hline Yes & $27(40)$ & $38(45)$ & 0.534 \\
\hline No & $41(60)$ & $47(55)$ & \\
\hline \multicolumn{4}{|l|}{ Wear sunglasses on sunny days } \\
\hline Yes & $27(40)$ & $45(53)$ & 0.103 \\
\hline No & $41(60)$ & $40(47)$ & \\
\hline \multicolumn{4}{|l|}{ Hours a day spent in open air } \\
\hline$<5$ hours & $46(68)$ & $52(61)$ & 0.668 \\
\hline $5-10$ hours & $20(29)$ & $29(34)$ & \\
\hline$>10$ hours & $2(3)$ & $4(5)$ & \\
\hline \multicolumn{4}{|l|}{ Clothing preferred in summer } \\
\hline Short-sleeved or sleeveless & $46(67)$ & $49(58)$ & 0.205 \\
\hline Long-sleeved & $22(32)$ & $36(42)$ & \\
\hline \multicolumn{4}{|c|}{ Clothing preferred when swimming } \\
\hline Swimsuit & $39(87)$ & $49(91)$ & 0.748 \\
\hline UV filter clothing & $6(13)$ & $5(9)$ & \\
\hline \multicolumn{4}{|l|}{ Use sunscreen when swimming } \\
\hline Yes & $24(53)$ & $34(63)$ & 0.333 \\
\hline No & $21(47)$ & $20(37)$ & \\
\hline \multicolumn{4}{|l|}{ Reapply sunblock after swimming } \\
\hline Yes & $12(27)$ & $15(28)$ & 0.902 \\
\hline No & $33(73)$ & $39(72)$ & \\
\hline \multicolumn{4}{|c|}{ Avoid sun 11 am to $4 \mathrm{pm}$ in summer } \\
\hline Yes & $42(62)$ & $61(72)$ & 0.190 \\
\hline No & $26(38)$ & $24(28)$ & \\
\hline
\end{tabular}

Table 4 | Knowledge and beliefs concerning disease severity and treatment status.

\begin{tabular}{|c|c|c|c|c|c|c|}
\hline Belief & $\begin{array}{c}\text { Focal } \\
\text { disease, } \\
n(\%)\end{array}$ & $\begin{array}{c}\text { Generalized } \\
\text { disease, } \\
n(\%)\end{array}$ & $p$-value & $\begin{array}{l}\text { Phototherapy } \\
\text { received, } \\
n(\%)\end{array}$ & $\begin{array}{c}\text { Phototherapy } \\
\text { not received, } \\
n(\%)\end{array}$ & $p$-value \\
\hline Sun makes my disease more visible & $51(75)$ & $76(89)$ & 0.032 & $54(87)$ & $73(80)$ & 0.372 \\
\hline Sun exacerbates my disease & $40(59)$ & $57(67)$ & 0.293 & $39(63)$ & $58(64)$ & 0.916 \\
\hline Sun improves my disease & $2(3)$ & $4(5)$ & 0.889 & $4(7)$ & $2(2)$ & 0.183 \\
\hline $\begin{array}{l}\text { I must protect myself from sun because of } \\
\text { my disease }\end{array}$ & $61(90)$ & $80(94)$ & 480 & $59(95)$ & $82(90)$ & 0.404 \\
\hline $\begin{array}{l}\text { I have a greater risk of skin cancer because } \\
\text { of my disease }\end{array}$ & $30(44)$ & $40(47)$ & 0.717 & $31(50)$ & 39 (43) & 0.384 \\
\hline $\begin{array}{l}\text { I have a lower risk of sunburn because } \\
\text { of my disease }\end{array}$ & $45(66)$ & $64(75)$ & 0.216 & $50(81)$ & $59(65)$ & 0.052 \\
\hline
\end{tabular}

at $86 \%$. Bhatia et al. (14) compared the sun-protection habits of 442 vitiligo patients, differentiating those that were members of a support group from those that were not. Rates of generalized disease and of exposure to UV for more than 1 hour a week were lower among patients that were not in a support group. The rate of belief that UV was good for the disease was also lower, although the history of sunburn in the previous year was higher. Sun protection was twice as high among members of a support group, and rates of sunscreen reapplication were also higher. Rates of sunscreen use in both groups (96-94\%) were much higher than in our study. Ahmet et al. reported that vitiligo patients experienced difficulties in maintaining good sun protection, such as the application of sunscreen (2).

Nearly half of our patients believed they were at increased risk of skin cancer because of their disease. Bhatia et al. reported a similar conclusion (14). Ahmed et al. reported severe anxiety due to fear of skin cancer among vitiligo patients, and they determined that some patients avoided the sun entirely (2). Unexpectedly, epidemiological studies of the association between vitiligo and skin cancer have determined a reduced cancer risk. Vitiligo is associated with polymorphism of the TYR gene, which codes tyrosinase, an essential enzyme in melanin synthesis. Different allelic connections may protect vitiligo patients from melanoma due to an anti-tyrosinase effect $(5,10,15)$. Autoimmunity is also thought to protect vitiligo patients against melanoma, although it has also been suggested that the lack of melanin can increase solar injury, as well as melanoma and $\operatorname{NMSC}(9,16)$. Only one of our patients had a history of skin cancer. Bhatia et al. (15) and Priska et al. (5) also observed no increased incidence of skin cancer in their patients. However, the mean age of the populations in our study and in the study by Priska et al. was markedly lower (5).

The photodamage and photoageing effects of PUVA and narrow-band UVB are thought to be responsible for increases in skin cancers. Teuling et al. (9) determined no increased risk for melanoma and NMSC in patients receiving phototherapy. In a study evaluating the sun-protection behaviors of patients that received phototherapy, Priska et al. (5) did not find any difference in the use of sunscreen, similar to our study, whereas in our study the rate of changing vacation habits and the preference for sunscreen with a high protection factor were higher.

We analyzed sun-protection habits in terms of age in our study; rates of changing vacation habits following diagnosis, using SPF 50 protection, wearing wide-brimmed hats, wearing sunglasses, and wearing long-sleeved clothing were all higher above the age of 18 , whereas use of sunscreen was higher among those under 18. Sun-protection habits in terms of age groups have not previously been analyzed in the literature.

Psychological effects such as embarrassment, lack of self-confidence, social anxiety, loss of self-esteem, and impaired quality of fidence, social anxiety, loss of self-esteem, and impaired quality of
life are well known in vitiligo patients $(2,5)$. One study highlighted 
that patients avoid outdoor activities. Patients have been shown to experience significant problems in exposing depigmented skin and therefore to feel a need to cover up, even in hot weather (2). In contrast, wearing short-sleeved and sleeveless clothing and swimsuits when swimming was not different from the general population in our study. In addition, no difference was determined in sun-protection habits when subjects with generalized disease and facial involvement were compared with other patients.

Our study also investigated patients' attitudes and beliefs concerning the effects of the sun on their disease. A significant proportion of our patients thought that sunlight made their disease more evident and exacerbated it. The belief that sunlight improved the disease was less common. Similarly, in Bhatia et al.'s study a significant proportion of patients thought that UV exposure made their disease more evident, although fewer thought that this exacerbated it (14).

The two previous studies had no healthy volunteer groups for comparison purposes. Ours is therefore the first study to compare sun-protection habits among vitiligo patients with the general population. A principal limitation of our study is the relatively small number of patients, and another is that $42 \%$ of patients were receiving active treatment.

\section{Conclusions}

Our findings showed that the most popular means of protection against the sun among vitiligo patients was to remain in the shade, followed by the use of sunscreen, sunglasses, widebrimmed hats, and long-sleeved clothing. We determined higher rates of sunscreen use, use of high-SPF products, and tendencies to remain in the shade in vitiligo patients compared to the general population. We also determined that the presence of generalized disease and of lesions in visible areas such as the face did not alter sun-protection habits. Although no relationship between vitiligo and skin cancer has been proved in the literature, half of our patients believed that it increased the risk of skin cancer.

\section{References}

1. Krüger C, Schallreuter KU. A review of the worldwide prevalence of vitiligo in Children/adolescents and adults. Int J Dermatol. 2012;51:1206-12.

2. Ahmed A, Steed L, Burden-Teh E, Shah R, Sanyal S, Tour S et al. Identifying key components for a psychological intervention for people with vitiligo-a quantitative and qualitative study in the United Kingdom using web-based questionnaires of people with vitiligo and healthcare professionals. J Eur Acad Dermatol Venereol. 2018;32:2275-83.

3. Wang Y, Li S, Li C. Perspectives of new advances in the pathogenesis of vitiligo: from oxidative stress to autoimmunity. Med Sci Monit. 2019;25:1017-23.

4. Mohammed GF, Gomaa AH, Al-Dhubaibi MS. Highlights in pathogenesis of vitiligo. World J Clin Cases. 2015;3:221.

5. McDonald PB, Zapata L, Rodrigues M. Sunscreen habits and skin cancer rates in patients with vitiligo in Australia. Australas J Dermatol. 2018;59:346-8.

6. Taieb A, Picardo M. Vitiligo. N Engl J Med. 2009;360:160-9.

7. Hexsel CL, Eide MJ, Johnson CC, Krajenta R, Jacobsen G, Hamzavi I, et al. Incidence of nonmelanoma skin cancer in a cohort of patients with vitiligo. J Am Acad Dermatol. 2009;60:929-33.

8. Gawkrodger DJ, Ormerod AD, Shaw L, Mauri-Sole I, Whitton ME, Watts MJ, et al. Guideline for the diagnosis and management of vitiligo. Br J Dermatol. 2008; 159:1051-76.

9. Teulings HE, Overkamp M, Ceylan E, Nieuweboer-Krobotova L, Bos JD, Nijsten $T$, et al. Decreased risk of melanoma and nonmelanoma skin cancer in patients with vitiligo: a survey among 1307 patients and their partners. Br J Dermatol. 2013;168:162-71.
10. Paradisi A, Tabolli S, Didona B, Sobrino L, Russo N, Abeni D. Markedly reduced incidence of melanoma and nonmelanoma skin cancer in a nonconcurrent cohort of 10,040 patients with vitiligo. J Am Acad Dermatol. 2014;71:1110-6.

11. Schallreuter KU, Tobin DJ, Panske A. Decreased photodamage and low incidence of non-melanoma skin cancer in 136 sun-exposed Caucasian patients with vitiligo. Dermatology. 2002;204:194-201.

12. Skin Cancer Foundation. Prevention guidelines [Internet]. [Cited 22 February 2016]. Available from: http://www.skincancer.org/prevention/sunprotection/ preventionguidelines.

13. Glanz K, Yaroch AL, Dancel M, Saraiya M, Crane LA, Buller DB, et al. Measures of sun exposure and sun protection practices for behavioral and epidemiologic research. Arch Dermatol. 2008;144:217-22.

14. Bhatia B, Kechichian E, Eleftheriadou V, Castelneau JP, Seité S, Meurant JM, et al. Habits and risk perception associated with sun exposure in vitiligo patients according to their participation in a patients' organization. J Eur Acad Dermatol Venereol. 2019;33: e100-3.

15. Schallreuter KU, Behrens-Williams S, Khaliq TP, Picksley SM, Peters EM, Marles LK, et al. Increased epidermal functioning wild-type p53 expression in vitiligo. Exp Dermatol. 2003;12:268-77.

16. Newton-Bishop JA, Chang YM, Elliott F, Chan M, Leake S, Karpavicius B, et al. Relationship between sun exposure and melanoma risk for tumours in different body sites in a large case-control study in a temperate climate. Eur J Cancer. 2011;47:732-41. 